\title{
Antibiogram of Aerobic Bacterial Isolates from Surgical Site Infections in a Tertiary Care Hospital in North India
}

\author{
Perika*, Suharshi Gupta and Shashi Sudhan Sharma \\ Department of Microbiology, GMC Jammu, India \\ *Corresponding author
}

\section{A B S T R A C T}

\section{Keywords}

Polymyxin B,

Klebsiella,

Pseudomonas,

Acinetobacter sp.,

Vancomycin

\section{Article Info}

Accepted:

18 August 2019

Available Online:

10 September 2019
Surgical site infections (SSIs) are an important cause of mortality and morbidity Worldwide, especially common in low and middle income countries. Prevalence of these infections ranges between 5-16\%, contributing to $20 \%$ of health care associated infection. Their source may be exogenous or endogenous. These infections are responsible for increasing the treatment cost, length of hospital stay, hence, a knowledge of the rate of SSIs can help in formulating appropriate policies for timely and accurate management in hospital. A vast majority of microbes present in hospital environment serve as reservoirs for these infections. The present study was done on 450 samples received in Microbiology dept of GMC Jammu over a period of 1 year (May 2018- May 2019) to determine the bacterial isolates causing SSIs and study their antibiogram. 420 samples (93.3\%) showed pure isolates while 20 cases $(4.44 \%)$ were of mixed infection. Most common isolates among Gram negative were Escherichia coli, Klebsiella, Pseudomonas, Acinetobacter sp. while Gram positive isolates were Staphylococcus aureus and Enterococcus sp. Among gram negative isolates most effective drug was Polymyxin B and Imipenem, while among gram positive Linezolid and Vancomycin were most effective. It was seen that multi drug resistant organisms were very common in our hospital. Strict implementation of antibiotic policy and creating awareness through education programmes can help in combating rapid spread of drug resistance.

\section{Introduction}

Human skin is harboured by a wide range of bacterial species - they may be present in the nasopharynx, gastrointestinal tract and other parts of the body with little potential for causing disease, because of the presence of first line of defense within the body (1). Surgical site infection (SSI) is defined as an infection that develops 30 days after a surgical procedure or 1 year if an implant is placed and the infection is often related to surgery ${ }^{(2,3)}$ These infections previously known as postoperative wound infections are one of the most common infections in low and middle income countries. Prevalence of these infections ranges between $5-16 \%$ and they contribute to $20 \%$ of health care associated infection.

Depending on the depth of penetration of infection into the wounds, SSIs are divided 
into three types: superficial incisional, deep incisional, and organ/space (4). Whether a surgical site will get clinically infected depends on a number factors like virulence of the pathogen, adjuvant effects of microenvironment such as ischaemia or foreign body and innate and acquired host defenses. Some patient related factors include smoking, presence of diabetes while operation related factors include duration of surgery, type of surgery etc ${ }^{(5)}$. It is important that healthcare workers recognize the patients with various risk factors for acquiring SSIs so that timely intervention can be taken to minimize the risk of contamination. Even though surgery has made great advances in last 3 quarters, postoperative wound infection is the most common complication faced by surgeon since the advent of surgery and they have become an important cause of mortality and morbidity due to emergence of antimicrobial resistant pathogens. Etiology of surgical wound infections is also linked to various local factors like hematomas, seromas, suture material, poor surgical technique, degree of contamination, age, hygiene, and other associated diseases.

SSIs may be acquired either from exogenous or endogenous sources. Exogenous sources are present external to the patient such as patient care personnel, visitors, patient care equipment, medical devices, or the health care environment while endogenous sources are the body sites such as skin, nose, mouth, gastrointestinal tract or vagina that are usually inhabited by microorganisms. Incidence of SSIs differs widely between surgical procedures, hospitals, patients, and between surgeons ${ }^{(6,7)}$. A variety of microbes are present in hospital environment and they serve as reservoir for SSIs. Complications linked to SSIs include increased readmission rates, enhanced overall costs, increased length of hospital stay ${ }^{(8)}$.
The most common bacterial organisms isolated from surgical wound infections are Enterobacteriaceae, Staphylococcus aureus, Pseudomonas aeruginosa and Coagulase negative Staphylococcus, Streptococcus, Enterococcus species ${ }^{(9,10,11)}$. In the recent years an increased number of gram negative infections are being reported as a common cause of surgical infections. Various surveillance data have shown that the type of organisms causing SSIs have not changed over the past 10-15 years, but the proportion of different types of organisms has changed and this has been attributed to increasing acquity of surgical patients, increased number of immune compromised patients and increased use of broad spectrum antibiotics ${ }^{(12)}$. An increased number of SSIs are now being caused by antimicrobial resistant organisms as well as by atypical bacterial and fungal organisms ${ }^{(8)}$.

Due to the paucity of data from India, present study was done to know the various bacterial isolates associated with SSIs and their antimicrobial susceptibility pattern. This would help in selecting the most appropriate and timely treatment options for SSIs, which in turn would help in reducing the cost of treatment and morbidity associated with disease.

\section{Materials and Methods}

Study design: Prospective and observational study

Study period : 1 year (May 2018- May 2019)

\section{Procedure}

This study was done in bacteriology section, Department of Microbiology, Govt. Medical College Jammu. 
A total of 450 samples received in bacteriology section from different various surgical wards (Orthopedics, General Surgery, Obstetrics and Gynaecology) were included in the study.

Sample collection was conducted by medical officers in wards using commercially available sterile cotton swabs and following existing departmental guidelines. Two swabs per patient were collected after carefully cleaning wound with sterile water to prevent surface contamination. Samples were transported to microbiology department within one hour of collection to prevent drying of swabs.

Swabs were immediately inoculated on Blood agar, MacConkey agar, incubated at $37^{\circ} \mathrm{C}$ aerobically for 24 hours. A portion of second swab was used to prepare a direct Gram smear in order to have a presumptive idea of presence of any infection.

Next day culture plates were checked for growth and to identify. Growth obtained was subjected to Gram stain to check for colony morphology and a battery of standard biochemical tests were applied as per standardized protocols of our laboratory.

Antimicrobial sensitivity testing was carried out by Kirby-Baeur disk diffusion method on Mueller Hinton agar as per CLSI guidelines.

Different panels of antimicrobial agents for Gram -positive and Gram-negative bacteria were used as per Clinical Laboratory Standards Institute(CLSI) guidelines ${ }^{(12)}$

\section{Results and Discussion}

Out of 450 samples processed, $420(93.3 \%)$ showed pure growth. 20 cases (4.44) had mixed infection and no growth was obtained in 10 cases $(2.22 \%)$. [Figure 1 ]. Out of 420 isolates obtained, majority of bacteria obtained were gram negative bacteria $(80 \%)$ as compared to gram positive bacteria (20\%). Among the gram negative isolates, most common was Escherichia coli 100 (23.8 \%), followed by Klebsiella pneumonia 69 (16.4 $\%)$, Pseudomonas aeruginosa 64 (15.2 \%), Acinetobacter spp. 53 (12.6\%), Citrobacter sp 20(4.7 \%), Proteus spp. 16 (3.8\%), Enterobacter 8 (1.9\%). [Table 1]

The microbiological profile of Gram positive organisms consisted of Staphylococcus aureus 50 (11.9\%), of which 10 cases were MRSA strains followed by Enterococcus sp 34 $(8.09 \%)$. Budding yeast was found in 6 cases $(1.42 \%)$. [Table 1 ]

Antimicrobial profile of most of gram negative isolates showed maximum sensitivity to Polymyxin B, Imipenem and Chloramphenicol while the most resistant drugs were Ampicillin, Third generation cephalosporins and amoxyclav. [Table 3]

Among the gram positive isolates most sensitive drugs were Linezolid, Vancomycin, Chloramphenicol while maximum resistance was seen with Penicillin, Ampicillin, Erythromycin. [Table 2]

Cases of mixed infection were 20 in number and most common infection was a combination of a gram positive and a gram negative organism like Staphylococcus+ Escherichia coli, Proteus+ Enterococcus, Staphylococcus + Acinetobacter sp., Escherichia coli+ Streptococcus.

Despite great advances made in the field of surgical techniques and understanding pathogenesis of wound infections, SSIs are an important cause of morbidity and mortality especially in developing countries ${ }^{(13,14)}$. Low rates of infection in developed countries may be due to vast differences in working conditions prevailing in these countries. 
Patients with SSI are twice as likely to die, $60 \%$ more likely to be admitted to the intensive care unit and more than five times more likely to be readmitted to the hospital after discharge. Management of these SSIs is a significant concern for physicians and surgeons in a health care facility. Hospital environment harbours a variety of pathogenic microbes which serve as a reservoir for the development of SSIs ${ }^{(15)}$. The widespread emergence of resistance to a wide array of antibiotics further contributes to the existing problem $^{(16)}$.

The rate of SSIs varies widely among different hospitals as well as worldwide. As per different studies the rate varies from $2.5 \%$ to $41.9 \%(17,18,19,20)$. As per our study rate of infection was found to be $25 \%$.

A number of local factors responsible for these infections include haematomas, seromas, suture material, poor surgical technique, degree of contamination, age, nutrition, hygiene. The rate of SSIs usually increases with the increase in age. This is due to poor immune response, presence of co morbidities, reduced compliance with treatment ${ }^{(19)}$. A prolonged pre -operative hospital stay with exposure to the hospital environment increases the risk of SSI ${ }^{(21)}$. Comparable findings were found in the present study.

A prolonged hospital stay leads to colonization with antimicrobial resistant microbes and affects patient's susceptibility to infection by lowering host resistance and increasing the opportunity for bacterial colonization (19). Also, administration of preoperative antibiotics is known to decrease the incidence of SSI (22,23). Prophylactic antibiotic usage was not a routine in our hospital. Only selected patients with some infection or other risk factors received antibiotic prophylaxis. The lack of attention towards the infection control measures, inappropriate hand hygiene practices and overcrowded hospitals are the major factors responsible for high rates of infection in the Indian hospitals ${ }^{(16)}$.

In the present study Staph aureus was the most common isolate among gram positive and this finding was consistent with the reports from other studies (Mundana, Bhave) [24,25]. The source of infection with Staphylococcus aureus is usually endogenous as it forms a part of normal flora of skin as well as nasal flora.

However infection can also be acquired from exogenous sources like contamination from environment, surgical instruments or hands of health care workers ${ }^{(26)}$.

The most effective drugs against Staph aureus were Linezolid, Vancomycin, Cotrimoxazole. This finding is of clinical importance as it can help in formulation of antibiotic policy of our hospital. In this study gram negative infections $(80 \%)$ were more common than gram positive $(20 \%)$.

This is in concordance with studies by $\mathrm{M}$. Saleem, et al., and Gelaw, et al., ${ }^{(27,28)}$. A high incidence of gram negative infections is often attributed to patient's normal endogenous microflora ${ }^{(25)}$. In this study most common isolates were Escherichia coli 100 (23.8 \%), followed by Klebsiella pneumonia 69 (16.4 $\%)$, Pseudomonas aeruginosa 64 (15.2 \%), Acinetobacter spp. 53 (12.6\%), Citrobacter sp 20(4.7 \%), Proteus spp. 16 (3.8 \%), Enterobacter 8 (1.9\%). [Table 1].

Similar observations were reported by other authors also (Lubega A, et al., and Kikkeri $\mathrm{NV}$, et al., $(29,30)$. Among gram negative isolates maximum sensitivity was seen with Polymyxin B, Imipenem, and Chloramphenicol. 
Table.1- Characterization of various isolates obtained from patients with surgical site infections

\begin{tabular}{|l|c|c|}
\hline Organism & Number of isolates & $\begin{array}{c}\text { Percentage of isolates } \\
(\mathbf{n = 4 2 0})\end{array}$ \\
\hline Escherichia coli & 100 & 23.8 \\
\hline Klebsiella pneumoniae & 69 & 16.4 \\
\hline Pseudomonas aeruginosa & 64 & 15.2 \\
\hline Acinetobacter $\boldsymbol{s p}$ & 53 & 12.6 \\
\hline Staphylococcus aureus & 50 & 11.9 \\
\hline Enterococcus $\boldsymbol{s p}$ & 34 & 8.09 \\
\hline Citrobacter & 20 & 4.7 \\
\hline Proteus & 16 & 3.8 \\
\hline Enterobacter & 8 & 1.9 \\
\hline Budding yeast & 6 & 1.42 \\
\hline
\end{tabular}

Table.2 Antibiotic sensitivity pattern of aerobic gram positive bacterial isolates in surgical site infections (Percentage of sensitive isolates)

\begin{tabular}{|l|c|c|}
\hline Antibiotics & $\begin{array}{c}\text { Staphylococcus aureus } \\
(\mathbf{n = 5 0})\end{array}$ & $\begin{array}{c}\text { Enterococcus sp } \\
(\mathbf{n = 3 4 )}\end{array}$ \\
\hline Penicillin & $0 \%$ & $0 \%$ \\
\hline Oxacillin & $0 \%$ & $0 \%$ \\
\hline Ampicillin & Not tested & $0 \%$ \\
\hline Cefoxitin & $60 \%$ & NT \\
\hline Ciprofloxacin & $50 \%$ & $20 \%$ \\
\hline Cotrimoxazole & $78 \%$ & NT \\
\hline Erythromycin & $40 \%$ & $35.2 \%$ \\
\hline Azithromycin & $60 \%$ & NT \\
\hline Gentamycin & $50 \%$ & $70 \%$ \\
\hline Vancomycin & $100 \%$ & $100 \%$ \\
\hline Linezolid & $100 \%$ & $100 \%$ \\
\hline Chloramphenicol & $92 \%$ & $85 \%$ \\
\hline
\end{tabular}

Fig.1 Demonstrating type of growth from swabs

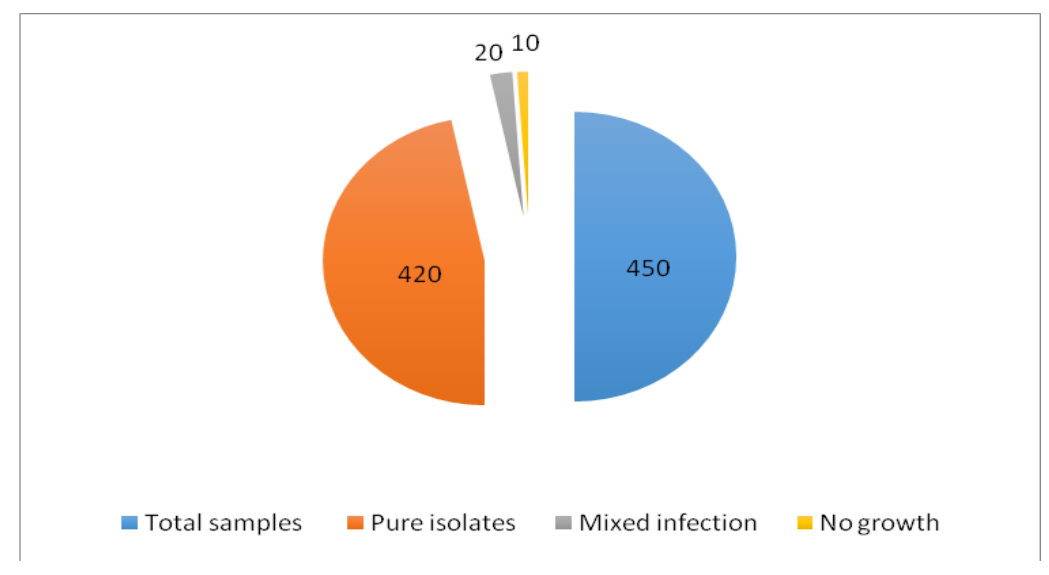


Table.3 Antibiotic sensitivity pattern of aerobic gram negative bacterial isolates in surgical site infections (Percentage of sensitive isolates)

\begin{tabular}{|l|c|c|c|c|c|c|}
\hline Antibiotics & $\begin{array}{c}\text { Escherichia coli } \\
(\mathbf{n = 1 0 0})\end{array}$ & $\begin{array}{c}\text { Klebsiella } \\
\text { pneumonia } \\
(\mathbf{n = 6 9})\end{array}$ & $\begin{array}{c}\text { Pseudomonas } \\
\text { aeruginosa } \\
(\mathbf{n = 6 4})\end{array}$ & $\begin{array}{c}\text { Proteus } \\
(\mathbf{n = 1 6})\end{array}$ & $\begin{array}{c}\text { Citrobacter } \\
(\mathbf{n = 2 0})\end{array}$ & $\begin{array}{c}\text { Acinetobacter } \\
(\mathbf{n = 5 3})\end{array}$ \\
\hline Ampicillin & $12 \%$ & $0 \%$ & $\mathrm{NT}$ & $\mathrm{NT}$ & $0 \%$ & $0 \%$ \\
\hline Amoxyclav & $31 \%$ & $2 \%$ & $\mathrm{NT}$ & $\mathrm{NT}$ & $0 \%$ & $1 \%$ \\
\hline Piperacillin- Tazobactum & $\mathrm{NT}$ & $\mathrm{NT}$ & $40 \%$ & $\mathrm{NT}$ & $\mathrm{NT}$ & $\mathrm{NT}$ \\
\hline Cefuroxime & $20 \%$ & $14 \%$ & $15 \%$ & $12 \%$ & $13 \%$ & $10 \%$ \\
\hline Cefotaxime & $32 \%$ & $16 \%$ & $14 \%$ & $14 \%$ & $10 \%$ & $11 \%$ \\
\hline Ceftazidime & $\mathrm{NT}$ & $\mathrm{NT}$ & $39 \%$ & $\mathrm{NT}$ & $\mathrm{NT}$ & $\mathrm{NT}$ \\
\hline Cefepime & $10 \%$ & $8 \%$ & $13 \%$ & $11 \%$ & $10 \%$ & $8 \%$ \\
\hline Imipenem & $90 \%$ & $95 \%$ & $93 \%$ & $90 \%$ & $92 \%$ & $97 \%$ \\
\hline Amikacin & $70 \%$ & $68 \%$ & $70 \%$ & $86 \%$ & $80 \%$ & $88 \%$ \\
\hline Polymyxin B & $100 \%$ & $100 \%$ & $100 \%$ & $100 \%$ & $100 \%$ & $100 \%$ \\
\hline Chloramphenicol & $95 \%$ & $93 \%$ & $\mathrm{NT}$ & $80 \%$ & $89 \%$ & $89 \%$ \\
\hline Doxycycline & $82 \%$ & $80 \%$ & $75 \%$ & $\mathrm{NT}$ & $\mathrm{NT}$ & $\mathrm{NT}$ \\
\hline
\end{tabular}

The emergence of multi drug resistant organisms from SSIs is of great concern. Maximum isolates from Enterobacteriaceae family were resistant to ampicillin, amoxicillin- clavulanic acid and even third generation cephalosporins. Various Indian and South East Asian workers have reported similar findings ${ }^{(31,32,)}$.

Nonfermenting gram negative bacilli are ubiquitous and notoriously multidrug resistant. In the present study $>60 \%$ of isolates of Pseudomonas were resistant to piperacillintazobactam, ceftazidime, cefepime. Such resistance patterns are attributed to injudicious use of antimicrobial agents and in such cases combination therapy along with meticulous wound care and barrier nursing precautions are required to avoid the spread of these bugs. Various Indian workers have reported similar findings ${ }^{(32)}$. The drug susceptibility findings of the present study show that antibiotics like third generation cephalosporins, ampicillin, amoxyclavulanic acid would be least useful for the treatment of infections caused by Enterobacteriaceae. Infections caused by non fermenters are challenging to treat as they are reported to be highly resistant, and due to their ability to survive easily and form biofilms. Also, it is known that perioperative prophylaxis can decrease the incidence of wound infection, isolation of such multidrug resistant strains is a cause of concern due to a limited repertoire of available antimicrobial agents.

The present study highlighted major bacterial flora isolated from SSIs in our hospital and their antibiotic susceptibility patterns.

This study would help clinicians in deciding appropriate antimicrobial therapy in order to control the rate of SSIs in our hospital. A proper knowledge of antibiotic susceptibility pattern is of utmost importance to decrease the spread of multi drug resistant organisms. Our study showed that majority of SSIs were resistant to multiple antibiotics.

Based on our findings various protocols which can be followed to decrease incidence of SSIs includes- hand hygiene and thorough infection control practices to reduce the spread of microbes and plasmid mediated drug resistance, antibiotic stewardship and prophylactic policies based on local susceptibility data, the maintenance of the infrastructure of operation theatre and wards, and finally - a close collaboration between microbiologists and surgeons ${ }^{(33)}$. 


\section{References}

Adwan G, Hasan NA, Sabra I, Sabra D, AlButmah S, Odeh S, et al., Detection of bacterial pathogens in surgical site infections and their antibiotic sensitivity profile. Int J Med Res Health Sci., 2016; 5(5): 75-82.

Anderson DJ, Sexton DJ, Kanafani ZA, Auten G, Kaye KS. Severe surgical site infection in community hospitals: Epidemiology, key procedures, and the changing prevalence of methicillin- resistant Staphylococcus aureus. Infect Control Hosp Epidemiol 2007; 28:1047-53.

Anvikar A, Deshmukh A, Karyakarte R, Damle A, Patwardhan N, Malik A, et al., One year prospective study of 3280 surgical wounds. Indian J Med Microbiol 1999;17:129-32.

Anvikar AR, Deshmukh AB, Karyakarte RP, Damle AS, Patwardhan NS, Malik AK, et al., 1999. A one year prospective study of 3280 surgical wounds. Indian $\mathrm{J}$ Med Microbiol.;17:129-32.

April 2013 CDC/NHSN Protocol Corrections, Clarification, and Additions. http://www.cdc.gov/nhsn/PDFs/pscManual/ 9pscSSI current.pdf (Accessed on July 10, 2013).

Barie PS. Surgical site infections: epidemiology and prevention. Surg Infect. 2002; 3(1):921.

Berard F, Gandon J. Factors infl uencing the incidence of wound infection. Ann Surg 1964;160:32-81.

Bhave PP, Kartikeyan S, Ramteerthakar MN, Patil NR. Bacteriological study of surgical site infections in a tertiary care hospital at Miraj, Maharashtra state, India. Int J Res Med Sci., 2016; 4(7): 2630-2635.

Budhani D, Kumar S, Sayal P, Singh S. Bacteriological profile and antibiogram of surgical site infection/ post-operative wound infection. International Journal of Medical Research and Review., 2016; 4(11): 19941999

CLSI. Performance standards for antimicrobial susceptibility testing; twentieth informational supplement CLSI document M100-S20 Wayne, PA: Clinical and Laboratory Standards Institute; 2010.
Desa LA, Sathe MJ. Factors infl uencing wound infection (a prospective study of 280 cases). JPostgrad Med1984;30:231-6.

Gelaw A, Gebre-Selassie S, Tiruneh M, Mathios E, Yifru S. Isolation of bacterial pathogens from patients with postoperative surgical site infections and possible sources of infections at the University of Gondar Hospital, Northwest Ethiopia. J Environ Occup Sci., 2014; 3(2): 103-108.

Kakati B, Kumar A, Gupta P, Sachan P, Thakuria B. Surgical site abdominal wound infections: Experience at a North Indian tertiary care hospital. Indian Academy of Clinical Medicine 2013;14:13-9.

Kirby JT, Sader HS, Walsh TR, Jones RN. Antimicrobial susceptibility and epidemiology of a worldwide collection of Chryseobacterium spp: Report from the SENTRY antimicrobial surveillance program (1997-2001). J Clin Microbiol 2004; 42:445-8.

Kumar A, Rai A. Prevalence of surgical site infection in general surgery in a tertiary care centre in India. International Surgery Journal 2017;4:3101-6.

Lilani SP, Jangale N, Chowdhary A, Daver GB. Surgical site infectionin clean and cleancontaminated cases. Indian J Med Microbiol 2005;23:249-52.

Lubega A, Joel B, and Lucy NJ. Incidence and Etiology of Surgical Site Infections among Emergency Postoperative Patients in Mbarara Regional Referral Hospital, South Western Uganda. Surgery Research and Practice, 2017.

Mahmood A. Bacteriology of surgical site infections and antibiotic susceptibility pattern of the isolates at a tertiary care hospital in Karachi. J Pak Med Assoc 2000; 50: 256-9.

Mangram AJ, Horan TC, Pearson ML, Silver LC, Jarvis WR. Guideline for prevention of surgical site infection, 1999. Hospital infection control practices advisory committee. Infect Control Hosp Epidemiol 1999; 20:250-78.

Mangram AJ, Horan TC, Pearson ML, Silver LC, Jarvis WR. Guideline for prevention of surgical site infection, 1999. Hospital 
Infection Control Practices Advisory Committee. Infect Control HospEpidemiol1999;20:250-78.

Mundhada AS, Tenpe S. A study of organisms causing surgical site infections and their antimicrobial susceptibility in a tertiary care government hospital. Indian $\mathbf{J}$ Pathol Microbiol., 2015; 58: 195-200.

Negi V, Pal S, Juyal D, Sharma MK, Sharma N. Bacteriological Profile of Surgical Site Infections and Their Antibiogram: A Study From Resource Constrained Rural Setting of Uttarakhand State, India. J Clin Diagn Res., 2015; 9(10): 17-20.

Ohalete, C. N., R. K. Obi, and M. C. EmeaKoroha, "Bacteriologyof different wound infection and their antimicrobial susceptibility patterns in Imo state Nigeria," World Journal of Pharmaceutical Sciences, vol. 13, no. 3, pp. 1155-1172, 2012.

Patel SM, Patel MH, Patel SD, Soni ST, Kinariwala DM, Vegad MM. Surgical site infections: Incidence and risk factors in a tertiary care hospital, Western India. Natl JCommunity Med 2012;3:193-6.

Pradhan GB, Agrawal J. Comparative study of post-operative wound infection following emergency lower segment caesarean section with and without the topical use of fusidic acid. Nepal Med Coll J., 2009; 11(3): 18991.

Reichman DE, Greenberg JA. Reducing Surgical Site Infections: A Review. Rev ObstetGynecol 2009;2:212-21.

Saleem M. Bacterial Profile and Antimicrobial Susceptibility. Pattern of Surgical Site Infections - A Retrospective Study. Indian Journal of Applied Research, 2015; 5(10): 204-206.
Sani, R.A. S.A.Garba, and O.A.Oyewole, Antibiotic resistance profile of gram negative bacteria isolated from surgical woundsin Minna, Bida, Kontagora and Suleja Areas of Niger State, American Journal of Medicine and Medical Sciences, vol. 2, no.1, pp. 20-24, 2012.

Setty NH, Nagaraja MS, Nagappa DH, Giriyaiah CS, Gowda NR, Laxmipathy Naik RD. A study on Surgical Site Infections (SSI) and associated factors in a government tertiary care teaching hospital in Mysore, Karnataka. Int J Med Public Health, 2014; 4: 171-5.

Sievert DM, Ricks P, Edwards JR, Schneider A, Patel J, Srinivasan A, et al., Antimicrobial-resistant pathogens associated with healthcare-associated infections: Summary of data reported to the national healthcare safety network at the centers for disease control and prevention, 2009-2010. Infect Control Hosp Epidemiol 2013; 34:1-4.

Suljagić V, Jevtic M, Djordjevic B, Jovelic A. Surgical site infections in a tertiary health care center: Prospective cohort study. Surg Today 2010;40:763-71.

Wong ES. Surgical site infections. In: Mayhall CG, editor. Hospital epidemiology and infection control. 1st ed. USA: Williams and Wilkins;1996.p.154-74.

Zhao, G., P. C. Hochwalt, M. L. Usui et al., "Delayed wound healing in diabetic $(\mathrm{db} / \mathrm{db})$ mice with Pseudomonas aeruginosa biofilm challenge: a model for the study of chronic wounds," Wound Repair and Regeneration, vol. 18, no. 5, pp. 467-477, 2010.

\section{How to cite this article:}

Perika, Suharshi Gupta and Shashi Sudhan Sharma 2019. Antibiogram of Aerobic Bacterial Isolates from Surgical Site Infections in a Tertiary Care Hospital in North India. Int.J.Curr.Microbiol.App.Sci. 8(09): 1517-1524. doi: https://doi.org/10.20546/ijcmas.2019.809.174 